\title{
Online Crystallography: \\ Automated, Remote Controlled Protein-to-Structure Pipelines for Drug Design
}

\author{
José A. Márquez \\ EMBL Grenoble, 71, Avenue des Martyrs, 38000 Grenoble, France \\ marquez@embl.fr
}

We have developed a novel approach called CrystalDirect that enables fully automated crystal mounting and cryo-cooling closing the automation gap between crystallization and X-ray data collection. The CrystalDirect technology also allows the automated delivery of small molecules to crystals, giving access to large scale small molecule screening through X-ray crystallography. We have combined this approach with automated data collection at the ESRF and other synchrotrons to develop a fully automated, remote-controlled pipelines for macromolecular crystallography and an automated pipeline for large scale compound and fragment screening to support structure guided drug discovery programs. In order to facilitate high throughput data analysis, we have built a series of Application Program Interfaces (APIs) linking the Crystallization Information Management System (CRIMS) and the ISPyB system for automated synchrotron data collection with automated structure refinement and analysis, using software pipelines developed by Global Phasing. These pipelines effectively provide online access to crystallization and synchrotron diffraction and data analysis facilities and remove key bottlenecks in modern crystallography. They can contribute to the rapid progression of challenging projects in structural biology, to facilitate the access to protein crystallography for scientist of other disciplines and stimulate translation of basic research into biomedical applications. On the other hand, the large amounts of data generated pose new challenges, but also provide new opportunities to develop integrated systems for data acquisition, processing and analysis. The experience from the use of these pipelines as well as the new opportunities enabled by the integration of crystallization, X-ray data collection and analysis into continuous, fully automated workflows will be discussed.

Keywords: Automation, High Throughput, Small molecule screening, Fragment screening, Data management and workflows, Drug design 